\title{
Bilateral thoracic Paravertebral block for immediate postoperative pain relief in the PACU: a prospective, observational study
}

\author{
Fei Liu ${ }^{1 *}$, HuanKai Zhang ${ }^{2}$ and Yunxia Zuo ${ }^{1}$
}

\begin{abstract} No complications related to bilateral TPVB were observed. after upper laparotomy in the PACU.

\section{Background}

Pain following laparotomy is a common complaint in the PACU. Good pain management improves participant satisfaction and facilitates shorter PACU/hospital stay [1]. Although pain management in the PACU has been improving with opioid-free strategies, opioids are still commonly used of analgesia for surgical participants with moderate to severe pain [2]. Intravenous opioids can provide rapid and effective analgesia but their undesired side effects, including pruritus, nausea and vomiting, urinary retention, and respiratory depression, result in discomfort or even lethal consequences [3, 4].
\end{abstract}

Background: To investigate the feasibility, effectiveness and safety of bilateral thoracic paravertebral block (TPVB) in the post anesthesia care unit (PACU) for pain relief in participants after laparotomy.

Methods: A single shot of bilateral TPVB with $25 \mathrm{ml}$ of $0.2 \%$ ropivacaine and $5 \mathrm{mg}$ dexamethasone in combination for both sides at the 8th thoracic transverse level (T8) was performed on 201 participants who complained moderate to severe pain on arrival to PACU after laparotomy. The visual analog scale (VAS) pain scores at rest and on cough, heart rate, blood pressure, and pulse oximetry before and after bilateral TPVB for up to $1 \mathrm{~h}$ were recorded. The VAS Pain scores at rest and on cough at $24 \mathrm{~h}$ after bilateral TPVB were also recorded.

Results: Bilateral TPVB was carried out successfully in all participants. The VAS pain scores at rest and on cough were $7.9 \pm 1.6$ and $8.7 \pm 1.3$ respectively pre-bilateral TPVB. The VAS pain scores at rest and on cough were significantly decreased to $1.1 \pm 1.2$ and $2.1 \pm 1.6$ respectively $(P<0.001)$ at $60 \mathrm{~min}$ after bilateral TPVB and to $2.1 \pm 1.7$ and $3.8 \pm$ 1.9 at rest and on cough respectively $((P<0.001)$ at $24 \mathrm{~h}$ after bilateral TPVB. At $10 \mathrm{~min}$ post-bilateral TPVB, only systolic blood pressure was reduced from $122 \pm 19 \mathrm{mmHg}$ to $111 \pm 18 \mathrm{mmHg}(P=0.007)$ but then gradually became stable.

Conclusion: Bilateral TPVB can be provided for pain relief to the participants who suffer from moderate to severe pain

Trial registration: Chinese Clinical Trial Registry: ChiCTR-ONN-16009229, Registered on 10 September 2016.

Keywords: Thoracic paravertebral block, Postoperative pain, Post anesthesia care unit

\footnotetext{
* Correspondence: 30333870@qq.com

'Department of Anesthesiology, West China Hospital, Sichuan University, No37 Guoxue Street, Chengdu, Sichuan 610041, People's Republic of China Full list of author information is available at the end of the article
}

TPVB is a technique of which local anesthetic is injected into the thoracic paravertebral space. The thoracic paravertebral space is a wedge-shaped space lying on either side of the vertebral column that fills with adipose tissue containing intercostal nerve, dorsal ramus, intercostal vessels, rami communicantes, and sympathetic trunk [5]. Therefore, TPVB in either side could produce ipsilateral, segmental, somatic and sympathetic nerve blockade in contiguous thoracic dermatomes $[5,6]$. It has been demonstrated that preoperative TPVB provides an excellent intraoperative and postoperative analgesia with less adverse effects in thoracic and abdominal surgery [7-12]. However, the effectiveness of TPVB as a rescue technique in the PACU for acute postoperative pain relief remains unknown. In this study, participants in the PACU who suffer moderate to severe pain after 
upper laparotomy were treated with a single-injection of local anesthetic via bilateral TPVB. Its effectiveness and safety profile were assessed prospectively.

\section{Methods}

The protocol was approved by the Ethics committee of West China Hospital, Sichuan University, China. Written informed consent was obtained from all participants prior to surgery. Participants were informed that they would be offered bilateral TPVB to be performed in the PACU if they reported moderate to severe pain which was not controlled adequately by the intraoperative and PACU analgesia that they had received. Inclusion criteria are: Age between 18 and 75 years; American Society of Anesthesiologists physical status 1 or 2; Laparotomy for hepatopancreatobiliary or gastrointestinal surgery; and participants with VAS pain score at rest $\geq 5$ in the PACU. Exclusion criteria: allergy to local anesthetics; spinal deformity; coagulation disorders; local infection at the injection site. Accordingly, total 359 participants were recruited into this study.

After arrived in the operation room, heart rate, noninvasive blood pressure, and pulse oximetry were monitored in all participants. General anesthesia was induced with intravenous midazolam $0.05 \mathrm{mg} / \mathrm{kg}$, sufentanyl $0.3-$ $0.5 \mu \mathrm{g} / \mathrm{kg}$, propofol $2-3 \mathrm{mg} / \mathrm{kg}$ and cis-atracurium $0.2 \mathrm{mg} / \mathrm{kg}$. Anesthesia was maintained with sevoflurane or propofol together with remifentanil $0.2-0.3 \mu \mathrm{g} / \mathrm{kg} / \mathrm{min}$. Analgesia consisted of Paricoxib $40 \mathrm{mg}$ administered $30 \mathrm{~min}$ before incision, and tramadol $100 \mathrm{mg}$ intravenously $30 \mathrm{~min}$ before the end of surgery. A patient control analgesia pump was started $30 \mathrm{~min}$ before the end of surgery, containing $1 \mu \mathrm{g} / \mathrm{ml}$ sufentanyl and $5 \mathrm{mg} / \mathrm{ml}$ tramadol, with a continuous infusion rate of $2 \mathrm{ml} / \mathrm{h}, 0.5 \mathrm{ml}$ bolus dose and lockout interval set to $15 \mathrm{~min}$. All participants were extubated at the end of surgery prior to transferring to the PACU. The VAS pain score at rest/cough and modified Aldrete score were assessed as soon as participants arrived in the PACU. Those 201 participants with the rest VAS $\geq 5$ and modified Aldrete score $\geq 9$ were finally enrolled in the study (Fig. 1).

Participants were placed in the left lateral decubitus position. A 2- to 5- MHz curved array transducer (M-Turbo, Sonosite, Bothell, USA) was used to scan from the midline laterally to identify the following anatomical landmarks: spinous process, transverse process, and the paravertebral space at the target vertebral level. The 8th thoracic transverse process was identified using ultrasound guidance from the 12th thoracic transverse process. An in-plane needle guidance technique with a $10 \mathrm{~cm}, 22 \mathrm{G}$ insulated needle (PAJUNK GmbH Medizin technologie, Geisingen, Germany) was used to perform the right lateral TPVB under aseptic conditions. After perforating the costotransverse ligament, $0.2 \%$ ropivacaine $25 \mathrm{ml}$ mixed with $5 \mathrm{mg}$ dexamethasone was injected after confirming a negative aspiration for blood. Anterior movement of the pleura indicated the appropriate spread of the local anaesthetic mixture in the paravertebral space (Fig. 2a, b). An out plane needle guidance technique was performed on the contralateral side using the same drug combination (Fig. 2c, d).

The VAS pain score at rest and on cough, heart rate, blood pressure, and pulse oximetry before and after bilateral TPVB for up to $1 \mathrm{~h}$ were recorded by every $10 \mathrm{~min}$. Hypotension was defined as a systolic blood pressure $<90 \mathrm{mmHg}$ within $1 \mathrm{~h}$ after bilateral TPVB. The VAS pain score at rest and on cough $24 \mathrm{~h}$ after bilateral TPVB were also recorded.

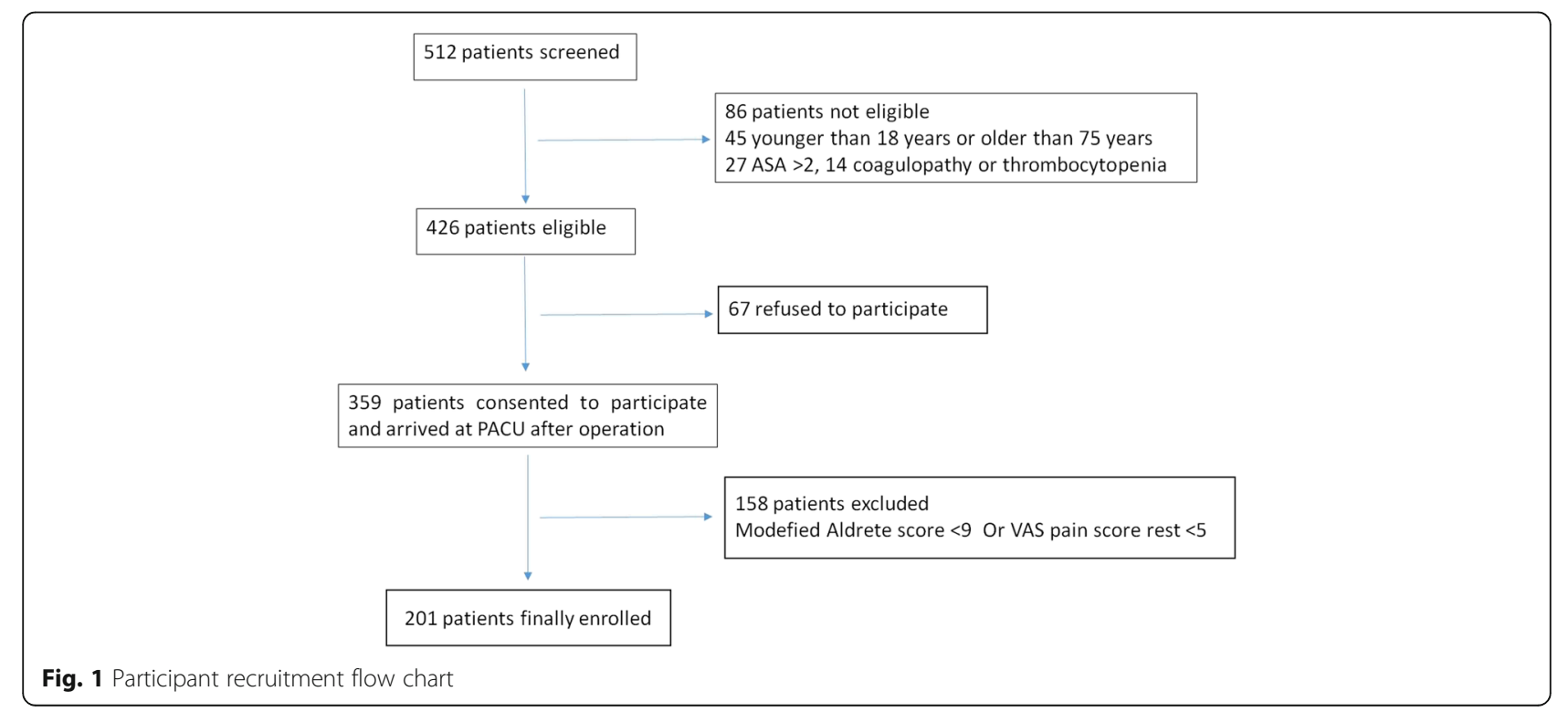




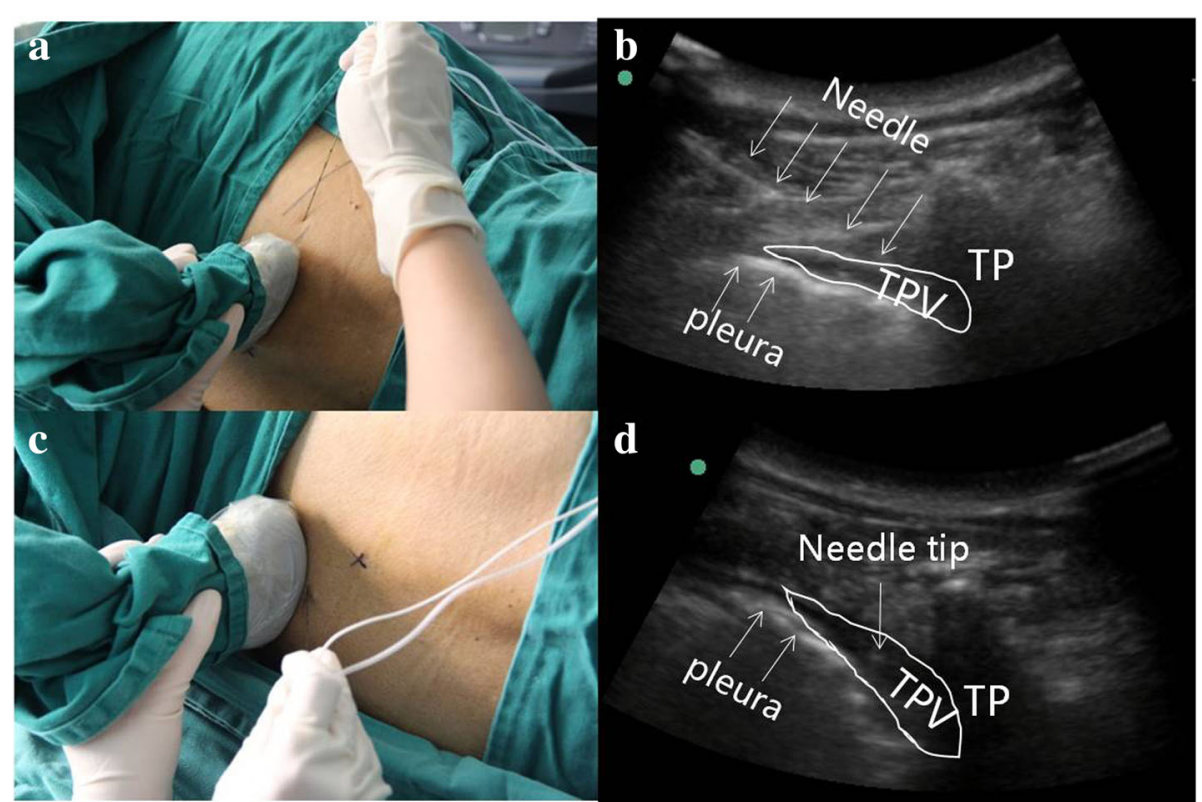

Fig. 2 Ultrasound guided baliteral thoracic paravertebral block at T8 level. $\mathbf{a}$ and $\mathbf{b}$ illustrating the plane needle insertion to the right T8 paravertebral space. $\mathbf{c}$ and $\mathbf{d}$ illustrating the plane needle insertion to the left T8 paravertebral space. TP $=$ Transverse process, TPV $=$ Thoracic paravertebral space

Statistical analyses were performed with SAS for (Windows, version 9.13). The quantitative data were presented as mean $\pm \mathrm{SD}$. The categorical data were presented as frequency and/or percentage. Binary logistic regression and multivariate logistic regression models were used to identify risk factors of hypotension after bilateral TPVB.

\section{Results}

Effective bilateral TPVB was achieved in 139 male and 62 female participants with age $52.8 \pm 12.1$ years and BMI $22.0 \pm 2.8 \mathrm{~kg} / \mathrm{m} 2$. The length of surgery was $178.9 \pm 62.7 \mathrm{~min}$. No complications associated with the bilateral TPVB (pneumothorax, pleural puncture, nerve injury, or vascular puncture) were observed in this study.
The VAS scores at rest and on cough were rapidly reduced from $7.9 \pm 1.6$ and $8.7 \pm 1.3$ of baseline before bilateral TPVB to $3.3 \pm 2.2$ and $4.2 \pm 2.310 \mathrm{~min}$ after bilateral TPVB respectively $(P<0.001)$ and then gradually decreased to $1.1 \pm 1.2$ and $2.1 \pm 1.6$ respectively $(P<0.001)$ at $1 \mathrm{~h}$ after injection (Fig. 3). No rescue analgesic was needed after bilateral TPVB in those patients after bilateral TPVB during their PACU stay. The VAS scores were $2.1 \pm 1.6$ and $3.8 \pm 1.9$ at rest and on cough $24 \mathrm{~h}$ after bilateral TPVB.

Systolic blood pressure was significantly decreased to $111 \pm 18 \mathrm{mmHg}(P<0.001)$ at $10 \mathrm{~min}$ after bilateral TPVB from the baseline of $123 \pm 19 \mathrm{mmHg}$. By definition, hypotension (systolic blood pressure $<90 \mathrm{mmHg}$ ) occurred in 24 patients $(11.9 \%)$. However, they were closely

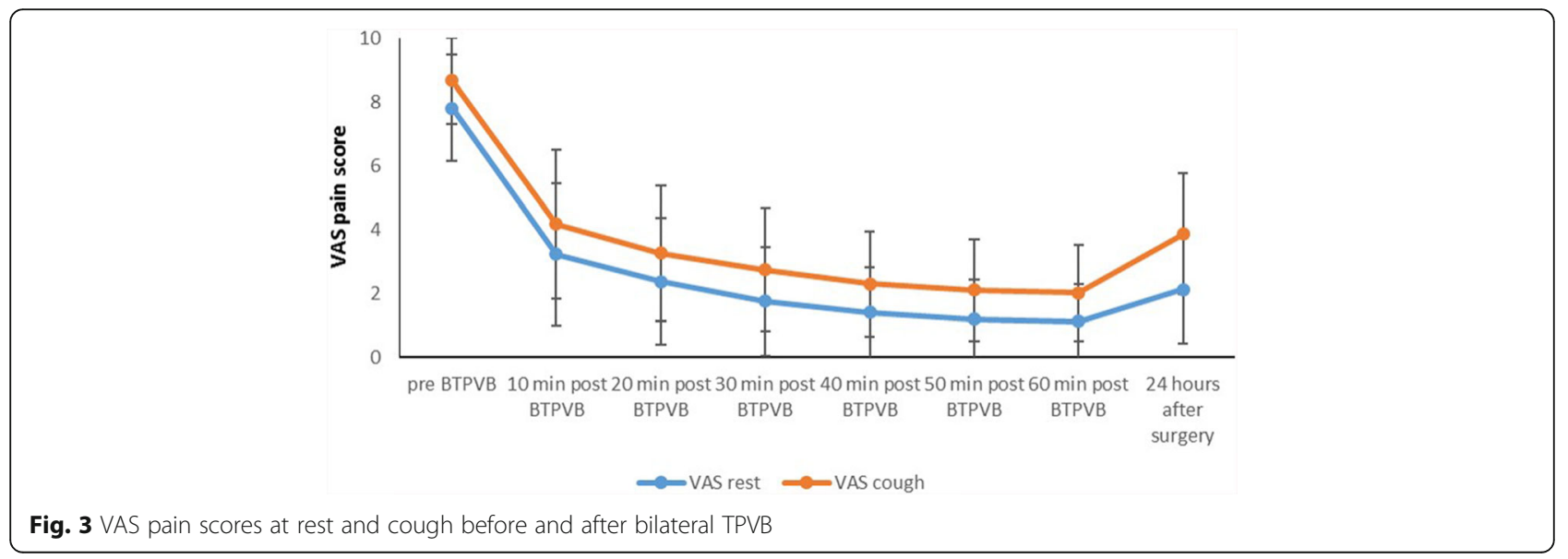




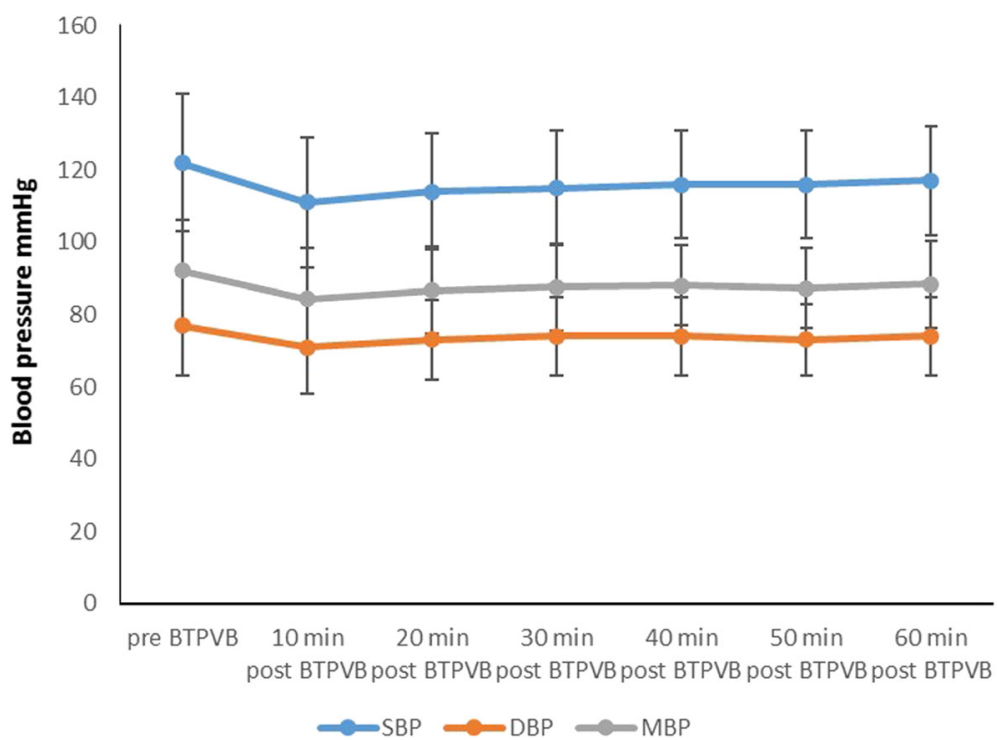

Fig. 4 The changes of systolic blood pressure, diastolic blood pressure and mean blood pressure before and post bilateral TPVB

monitored and their systolic blood pressure were gradually returned to the baseline within $60 \mathrm{~min}$ after bilateral TPVB (Fig. 4). With a binary logistic regression model analysis, body weight, operative time, systolic blood pressure, diastolic blood pressure and mean blood pressure lower readings before bilateral TPVB were risk factors for hypotension (Table 1). Using multivariate logistic regression model analysis, low systolic blood pressure before bilateral TPVB and heavy body weight were indentified to be independent risk factors for hypotension after bilateral TPVB (Table 2). Diastolic blood pressure was to be a similar pattern trend change as systolic blood pressure (Fig. 4). No significant changes of SPO2 and heart rate were found throughout the study (Fig. 5).

\section{Discussion}

Our results showed that ultrasound-guided bilateral TPVB is an effective rescue analgesic technique for moderate and severe pain which is not adequately controlled by conventional intravenous analgesia after upper laparotomy.

It has been reported that in thoracic surgeries, unilateral TPVB was as effective as epidural analgesia but with much less side effects [13, 14]. Furthermore, considerable evidence showed that TPVB in addition to GA provide a better postoperative pain control with fewer adverse effects when compared GA alone in video-assisted thoracoscopic surgery and breast surgery [15-17]. However, it was not often reported to be used for postoperative pain relief after laparotomy under GA. Richardson et al. have reviewed 541 participants undergoing bilateral TPVB in 12 various studies, and they concluded that bilateral TPVB is safe and effective for thoracic and abdominal surgery
[12]. However, the methods and outcomes in those studies are variable. Although many studies found that unilateral TPVB produced similar analgesia efficacy as compared with epidural block for thoracic surgery, a few studies have yet been compared bilateral TPVB with epidural analgesia for laparotomy which is generally considered to be a standard approach for postoperative pain relief after laparotomy. Recently Schreiber et al. found that there was modest analgesic advantage of thoracic epidural over bilateral TPVB for participants after open liver resection [18]. However, as the author also pointed out, technical risk and rare but serious

Table 1 Binary logistic regression model for risk factors of hypotension after bilateral TPVB

\begin{tabular}{lllll}
\hline Variable & $\begin{array}{l}\text { Hypotensive } \\
\text { group 24 }\end{array}$ & $\begin{array}{l}\text { Non-Hypotensive } \\
\text { group } 177\end{array}$ & P \\
\hline Sex(male/female) & $18 / 6$ & $120 / 57$ & 0.477 & 1.425 \\
Age & $51 \pm 11$ & $53 \pm 12$ & 0.465 & 0.987 \\
Body weight & $63.9 \pm 8.8$ & $58.8 \pm 9.4$ & $0.015^{*}$ & 1.059 \\
Height & $166.2 \pm 6.8$ & $164.1 \pm 7.4$ & 0.202 & 1.039 \\
BMI & $22.9 \pm 2.6$ & $21.8 \pm 2.8$ & 0.053 & 1.170 \\
Operative time & $205 \pm 49$ & $174 \pm 66$ & $0.030^{*}$ & 1.007 \\
Heart rate & $82 \pm 14$ & $80 \pm 14$ & 0.674 & 1.007 \\
Systolic blood pressure & $107 \pm 16$ & $125 \pm 19$ & $<0.001^{*}$ & 0.938 \\
Diastolic blood pressure & $71 \pm 15$ & $78 \pm 13$ & $0.011^{*}$ & 0.957 \\
Mean blood pressure & $83 \pm 14$ & $94 \pm 14$ & $0.001^{*}$ & 0.938 \\
VAS pain score rest & $7.6 \pm 1.9$ & $7.8 \pm 1.6$ & 0.618 & 0.937 \\
VAS pain score cough & $8.4 \pm 1.6$ & $8.7 \pm 1.3$ & 0.381 & 0.873 \\
\hline BMI Body Mass Index, VAS Visua & & &
\end{tabular}

BMI Body Mass Index, VAS Visual Analog Scale, ${ }^{*} P<0.05$ 
Table 2 Multivariate logistic regression model for risk factors of hypotension

\begin{tabular}{llllll}
\hline Variable & Estimate & StdErr & Wald & $P$ & OR \\
\hline BW & 0.066 & 0.026 & 6.357 & 0.012 & 1.068 \\
Systolic blood pressure & -0.066 & 0.017 & 15.567 & $<0.001$ & 0.936 \\
\hline BW Body Weight & & & & &
\end{tabular}

complications such as epidural hematoma, perioperative hypotension related to epidural block can not be ignored. Furthermore, Schreiber et al. used a traditional landmarkbased method for TPVB catheter insertion instead of ultrasound guidance or intraoperative placement by surgeons under direct vision, which may lead to more variable analgesic efficacy.

Instead of multiple injection at different thoracic level, a single injection of thoracic paravertebral block on each side at the T8 level was performed to reduce procedure time in a cohort of participants who were already in considerable pain. A previous study investigated the spread of local anesthetic in cadavers, and found no difference in paravertebral segment spread over 3-4 vertebral segments between a single versus dual-injection technique [19]. Our study showed that a single injection with large volume can provide good analgesic effect for large middle abdominal incision, indicating that multiinjections are not necessary.

Dynamic VAS scores at rest and on cough showed that bilateral TPVB provided effective analgesia with a rapid onset, supporting the use of bilateral TPVB in the PACU as an effective rescue analgesic technique. The rapid onset could be explained by the spinal nerve in the thoracic paravertebral space lacking both an epineurium and part of the perineurium, and with only a thin membranous root sheath. All these could enhance rapid local anaesthetic penetration, providing effect and rapid analgesia [20]. A mild decrease of systolic pressure without further treatment at $10 \mathrm{~min}$ after block and no changes of heart rate and $\mathrm{SPO}_{2}$ indicated that bilateral TPVB has minimal inhibitory effects on cardiorespiratory system.

Our study is limited as we assessed the efficacy of bilateral TPVB as a rescue technique for inadequate analgesia from our standard intravenous regimen, rather than comparing the efficacy of bilateral TPVB prior to surgery. A different study is required to test that research question. Furthermore, our study is observational without epidural block and or intravenous analgesic as the control groups and therefore we couldn't conclude if bilateral TPVB gives better pain relief and has less side effects. Lastly, only a single shot was conducted and therefore, it is not known how the picture of bilateral TPVB with continuous infusions by catheter insertion is when compared with a single shot injection. Anyhow, our study showed that bilateral TPVB under the aid of ultrasound guide at the T8 level in the PACU provides immediate and good analgesia for patients suffering moderate or severe pain after laparotomy without significant side effects. However, due to the nature of the observatory study reported here, clinical trials are needed to further confirm its clinical safety and effectiveness.

\section{Conclusions}

In summary, the use of ultrasound guided bilateral TPVB as a rescue analgesic technique in the PACU is effective and provides rapid onset of analgesia.

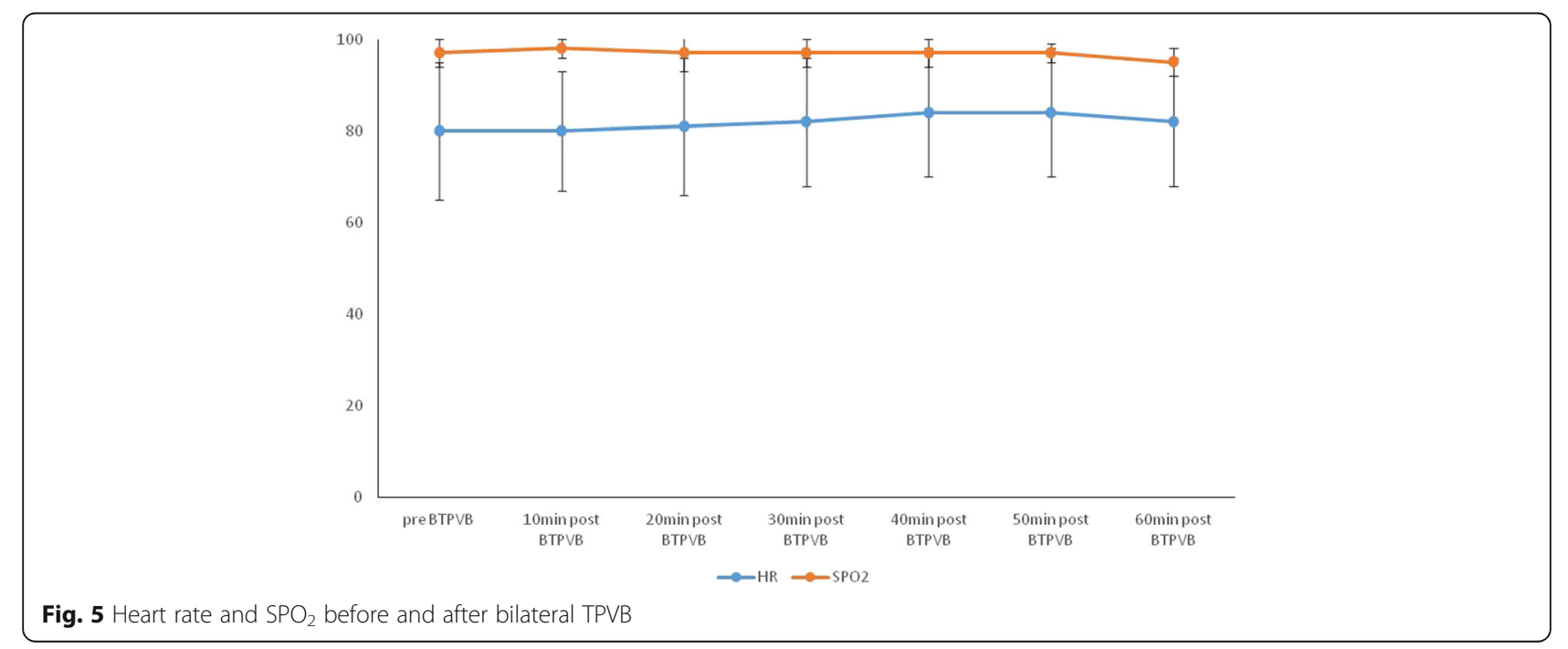




\section{Abbreviations}

PACU: Post anesthesia care unit; T8: 8th thoracic transverse level; TPVB: Thoracic paravertebral block; VAS: Visual analog scale.

\section{Acknowledgements}

The authors are grateful to the nurses in the PACU from the department of anesthesiology, West China Hospital, who contributed to this study.

\section{Funding}

This research was supported by a grant from the National Natural Science Foundation of China (No. 81601074).

\section{Availability of data and materials}

The raw data of the current study are available from the corresponding author on reasonable request.

\section{Authors' contributions}

FL conceived and designed the study, performed the experiments and drafted the manuscript. HZ collected the experimental data and assisted with data analysis. YZ conceived the study. All authors read and approved the final manuscript.

\section{Competing interests}

The authors declare that they have no competing interests.

\section{Consent for publication}

Not applicable.

\section{Ethics approval and consent to participate}

Ethics committee of West China Hospital approved this study under number 2015/09. An informed consent was presented to all patients prior to enrollment in the study group. Written informed consent to participate were obtained from all the participants.

\section{Publisher's Note}

Springer Nature remains neutral with regard to jurisdictional claims in published maps and institutional affiliations.

\section{Author details}

'Department of Anesthesiology, West China Hospital, Sichuan University, No37 Guoxue Street, Chengdu, Sichuan 610041, People's Republic of China. 2Department of Anesthesiology, Jie yang City people's Hospital, Jie yang, Guangdong 522000, People's Republic of China.

Received: 11 November 2016 Accepted: 13 June 2017

Published online: 05 July 2017

\section{References}

1. Bardiau FM, Taviaux NF, Albert A, Boogaerts JG, Stadler M. An intervention study to enhance postoperative pain management. Anesth Analg. 2003;96: 179-85.

2. Gandhi K, Baratta JL, Heitz JW, Schwenk ES, Vaghari B, Viscusi ER. Acute pain management in the postanesthesia care unit. Anesthesiol Clin. 2012;30:1-15.

3. Aubrun F, Mazoit JX, Riou B. Postoperative intravenous morphine titration. Br J Anaesth. 2012;108:193-201.

4. Ho KY, Gan TJ. Opioid-related adverse effects and treatment options. In: Sinatra RS, de Leon-Casasola OA, Ginsberg B, et al., editors. Acute pain management. New York: Cambridge University Press; 2009. p. 406-15.

5. Karmakar MK. Thoracic Paravertebral block. Anesthesiology. 2001;95:771-80.

6. Krediet AC, Moayeri N, van Geffen GJ, Bruhn J, Renes S, Bigeleisen PE, Groen GJ. Different approaches to ultrasound-guided thoracic Paravertebral block: an illustrated review. Anesthesiology. 2015;123:459-74.

7. Komatsu T, Kino A, Inoue M, Sowa T, Takahashi K, Fujinaga T. Paravertebral block for video-assisted thoracoscopic surgery: analgesic effectiveness and role in fast-track surgery. Int J Surg. 2014;12:936-9.

8. TerkawiAS, Tsang S, Sessler DI, Terkawi RS, Nunemaker MS, Durieux ME, Shilling A. Improving Analgesic Efficacy and Safety of Thoracic Paravertebral Block for Breast Surgery: A Mixed-Effects Meta-Analysis. Pain Physician. 2015; 18:757-80.
9. Baik JS, Oh AY, Cho CW, Shin HJ, Han SH, Ryu JH. Thoracic Paravertebral block for Nephrectomy: a randomized, controlled. Observer-Blinded Study Pain Med. 2014;15:850-6.

10. Marqués A, García R, Gómez F, Giammaria F. Ultrasound-guided paravertebral block for management of abdominal pain after transarterial embolization using drug-eluting beads loaded with irinotecan. J Vasc Interv Radiol. 2013;24:1416-7.

11. Chen H, Liao Z, Fang Y, Niu B, Chen A, Cao F, Mei W, Tian Y. Continuous right thoracic paravertebral block following bolus initiation reduced postoperative pain after right-lobe hepatectomy: a randomized, doubleblind, placebo-controlled trial. Reg Anesth Pain Med. 2014:39:506-12.

12. Richardson J, Lonnqvist PA, Naja Z. Bilateral thoracic paravertebral block: potential and practice. Br J Anaesth. 2011;106(2):164-71.

13. Ding X, Jin S, Niu X, Ren H, Fu S, Li Q. A comparison of the analgesia efficacy and side effects of Paravertebral compared with epidural blockade for Thoracotomy: an updated meta-analysis. PLoS One. 2014;9:e96233.

14. Yeung JH, Gates S, Naidu BV, Wilson MJ, Gao Smith F. Paravertebral block versus thoracic epidural for patients undergoing thoracotomy. Cochrane Database Syst Rev. 2016, 21;2:CD009121.

15. Matyal R, Montealegre-Gallegos M, Shnider M, Owais K, Sakamuri S, Shakil O, Shah V, Pawlowski J, Gangadharan S, Hess P. Preemptive ultrasound-guided paravertebral block and immediate postoperative lung function. Gen Thorac Cardiovasc Surg. 2015;63(1):43-8.

16. Amlong C, Guy M, Schroeder KM, Donnelly MJ. Out-of-plane ultrasoundguided paravertebral blocks improve analgesic outcomes in patients undergoing video-assisted thoracoscopic surgery. Local Reg Anesth. 2015; 15(8):123-8.

17. Schnabel A, Reichl SU, Kranke P, Pogatzki-Zahn EM, Zahn PK. Efficacy and safety of paravertebral blocks in breast surgery: a meta-analysis of randomized controlled trials. Br J Anaesth. 2010;105:842-52.

18. Schreiber KL, Chelly JE, Lang RS, Abuelkasem E, Geller DA, Marsh JW, Tsung A, Sakai T. Epidural versus Paravertebral nerve block for postoperative analgesia in patients undergoing open liver resection: a randomized clinical trial. Reg Anesth Pain Med. 2016;41(4):460-8.

19. Cowie B, McGlade D, Ivanusic J, Barrington MJ. Ultrasound-guided thoracic paravertebral blockade: a cadaveric study. Anesth Analg. 2010;110:1735-9.

20. Baik JS, Oh AY, Cho CW, Shin HJ, Han SH, Ryu JH. TPVB for nephrectomy: a randomized, controlled, observer-blinded study. Pain Med. 2014;15:850-6.

\section{Submit your next manuscript to BioMed Central and we will help you at every step:}

- We accept pre-submission inquiries

- Our selector tool helps you to find the most relevant journal

- We provide round the clock customer support

- Convenient online submission

- Thorough peer review

- Inclusion in PubMed and all major indexing services

- Maximum visibility for your research

Submit your manuscript at www.biomedcentral.com/submit
Biomed Central 